\title{
Generating high-brightness and coherent soft $x$-ray pulses in the water window with a seeded free-electron laser
}

\author{
Kaishang Zhou, Chao Feng, ${ }^{*}$ Haixiao Deng, and Dong Wang \\ Shanghai Institute of Applied Physics, Chinese Academy of Sciences, Shanghai 201800, China
}

(Received 4 September 2016; published 23 January 2017)

\begin{abstract}
We propose a new scheme to generate high-brightness and temporal coherent soft $\mathrm{x}$-ray radiation in a seeded free-electron laser. The proposed scheme is based on the coherent harmonic generation (CHG) and superradiant principles. A CHG scheme is first used to generate a coherent signal at ultrahigh harmonics of the seed. This coherent signal is then amplified by a series of chicane-undulator modules via the fresh bunch and superradiant processes in the following radiator. Using a representative of a realistic set of parameters, three-dimensional simulations have been carried out and the simulations results demonstrated that $10 \mathrm{GW}$-level ultrashort ( $20 \mathrm{fs}$ ) coherent radiation pulses in the water window can be achieved by using a $1.6 \mathrm{GeV}$ electron beam based on the proposed technique.
\end{abstract}

DOI: 10.1103/PhysRevAccelBeams.20.010702

\section{INTRODUCTION}

Intense, ultrashort, coherent soft $\mathrm{x}$-ray pulses generated by free-electron lasers (FELs) are becoming an essential tool for achieving significant breakthroughs in various scientific domains such as femtochemistry, material science, biology, and so on. Currently, most of the presently existing or planned x-ray FEL facilities [1-6] are based on the self-amplified spontaneous emission (SASE) principle $[7,8]$. While the radiation from a SASE FEL has always good spatial coherence, it typically has rather limited temporal coherence and large shot-to-shot fluctuations. There are a number of scientific applications, such as resonant scattering and spectroscopic techniques that require, or could benefit from, a better temporal coherence and high spectral brightness [9-12].

To improve the temporal properties, several SASE-based techniques have been developed in recent years [13-20]. However, the final output pulses from these schemes still suffer from large intensity or central wavelength fluctuations due to the energy jitter of the electron beam. For example, in the self-seeding scheme the energy fluctuations will translate into intensity FEL fluctuations, while in the HB-SASE scheme the energy fluctuations will affect the central wavelength stability. An alternative way to significantly improve the temporal coherence of SASE relies on the manipulation of the electron beam longitudinal phase space through seeding schemes using external laser

\footnotetext{
*Corresponding author. fengchao@sinap.ac.cn

Published by the American Physical Society under the terms of the Creative Commons Attribution 3.0 License. Further distribution of this work must maintain attribution to the author $(s)$ and the published article's title, journal citation, and DOI.
}

sources, such as high-gain harmonic generation (HGHG) [21], echo-enabled harmonic generation (EEHG) [22,23] or phase-merging enhanced harmonic generation [24,25]. Here after, we will be referring to the external seeding schemes simply as seeded FELs. Seeding the FELs with external coherent laser pulses has the additional advantage of providing radiation pulses with well-defined timing with respect to the seed laser, allowing pump-probe experiments to be performed with high temporal resolution. What is more, in a seeded FEL, one can manipulate the longitudinal phase correlation and control the relative phase of the radiation pulses, which is necessary for some phase-related experiments $[11,12]$.

One drawback of seeded FELs is that the wavelength cover range is limited due to the lack of suitable seeding laser at a short wavelength. For a commercial UV laser with a central wavelength around $260 \mathrm{~nm}$, the harmonic upconversion number of a single stage HGHG is significantly lower than what is required for reaching the $\mathrm{x}$-ray region [26]. Further extension of the output to the shorter wavelength needs a larger energy modulation amplitude, which will degrade the quality of the electron beam and results in a lower FEL output power in the following radiator. When the laser induced energy spread is too large, the FEL will work in the coherent harmonic generation $(\mathrm{CHG})$ regime [21], where the power amplification process will be quickly saturated and no exponential growth is expected in the radiator. To improve the frequency multiplication efficiency of a seeded FEL with a small laser induced energy spread, more complicated schemes such as cascaded HGHG or EEHG have been developed [27-31].

In this paper, we propose a more straightforward method, termed high-brightness HGHG (HB-HGHG), to significantly enhance the output peak power of a seeded FEL. The "brightness" here is defined as the number of photons 
per second emitted per unit surface area per unit solid angle within a bandwidth of $0.1 \%$ of the central wavelength. The proposed technique combines the $\mathrm{CHG}$ technique with the superradiant method [32-36]. First, an ultrashort coherent radiation pulse at the target harmonic of the seed laser is generated by a small part of the electron beam via the $\mathrm{CHG}$ process. Then this coherent signal is continually amplified by the fresh part of the electron beam in a long radiator with a series of temporal shifters to enhance the peak brightness of the FEL. Our method has the following advantages with respect to the previous seeded FEL schemes: (i) When compared with the conventional HGHG scheme, it has much higher harmonic up-conversion efficiency in a single stage setup, and thus holds the ability to produce x-ray radiation directly from a UV seed laser. (ii) When compared with the cascaded HGHG and EEHG schemes, it can simplify the overall design and provide much higher output brightness. The proposed scheme can be easily implemented at already existing or planned seeded FEL facilities, such as FERMI FEL [26,37] or the Shanghai soft X-ray FEL facility (SXFEL) [38,39].

This paper is organized as follows: Section II shows the layout and principle of the HB-HGHG. Section III discusses the requirements for the seed laser and the delay chicanes to realize the proposed technique. With realistic electron beam parameters, optimized start-to-end simulations for a soft x-ray FEL facility based on the HB-HGHG are given in Sec. IV. Finally, the conclusions are summarized in Sec. V.

\section{SCHEMATIC DESCRIPTION OF THE HB-HGHG}

Figure 1 shows the schematic layout of the proposed HB-HGHG scheme, which consists of a conventional CHG scheme and an amplifier with a series of chicane-undulator modules. An ultrashort UV seed laser pulse is adopted to interact with the tail part of the electron beam in the modulator (M) to generate a sinusoidal energy modulation [Fig. 1(a)]. This energy modulation is then converted into an associated density modulation by the dispersion section (DS) and subsequent coherent emission at a target high harmonic of the seed can be achieved in the following short radiator $(R 1)$, as shown in Fig. 1(b). The density modulation of the electron beam can be quantified by the bunching factor, which has a maximal value of unity. The $n$th harmonic bunching factor can be derived as [21]

$$
b_{n}=J_{n}(n D A) \exp \left(-\frac{n^{2} D^{2}}{2}\right)
$$

where $J_{n}$ is the $n$th order first class Bessel function, $D=k_{s} R_{56} \sigma_{\gamma} / \gamma$ is the dimensionless parameter related to the dispersive strength of the DS, $k_{s}$ is the wave number of the seed laser, $R_{56}$ is the momentum compaction generated by the DS, $\gamma$ is the Lorentz factor, $\sigma_{\gamma}$ is the initial uncorrelated energy spread, $A=\Delta \gamma / \sigma_{\gamma}$, and $\Delta \gamma$ represents the energy modulation amplitude induced by the seed laser. To optimize the $n$th harmonic bunching factor, the product of $n$ and $D$ in the exponential term of Eq. (1) should be small enough to limit the energy spread effect, while the product of $n, D$, and $A$ should be optimized to give a considerable value of $J_{n}$. Here we assume $n D<1$ and want $J_{n}$ to reach its first and absolute maximum; the requirement is $n D A \approx n+1$, which means that $\Delta \gamma$ should be approximately $n$ times larger than $\sigma_{\gamma}$. Therefore, for a large harmonic number, one also needs a large energy modulation amplitude. However, when the laser induced energy spread is too large, e.g. larger than the Pierce parameter $\rho_{r}$ [8], there will be no exponential growth of the FEL, resulting in very low output power. Therefore the need to limit the growth of the energy spread prevents the possibility of reaching a short wavelength in a single stage HGHG.

The FEL gain process in the radiator of HGHG can be divided in to three stages: the CHG with quadratic growth, the exponential growth, and saturation. In the first two gain lengths of the radiator, the FEL works in the CHG regime, where the harmonic field grows linearly with the distance

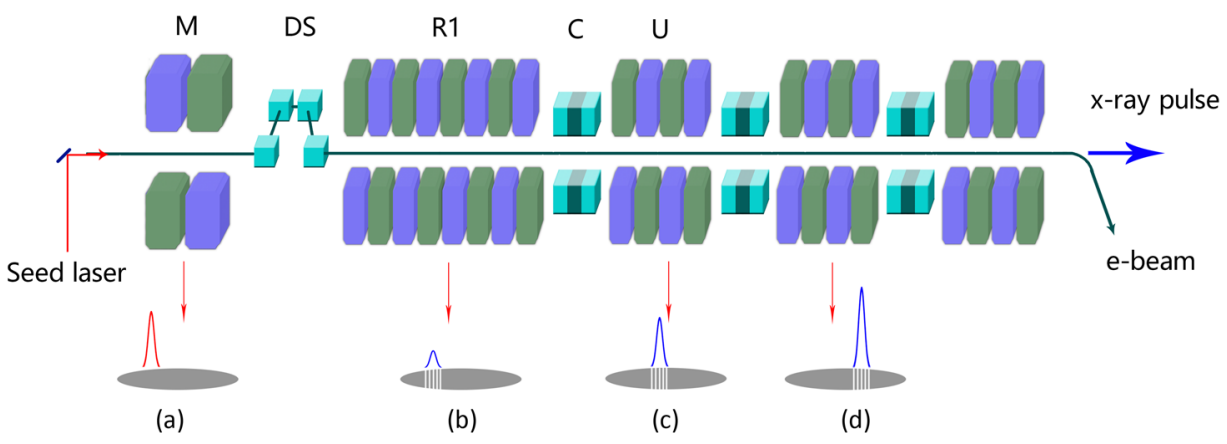

FIG. 1. Schematic layout of the HB-HGHG scheme. (a) A high power seed laser (red) is used to introduce the energy modulation into the tail part of the electron beam in the modulator (M). (b) The energy modulation is converted into density modulation by the dispersion section (DS). The first radiator section ( $R 1)$ is used to generate coherent signal (blue) at a target high harmonic of the seed. (c),(d) The following radiator that consists of chicane-undulator $(\mathrm{C}-\mathrm{U})$ modules are used to amplify the coherent signal (blue). 
traversed in the radiator $z$, and the peak power grows as $z^{2}$. For a longitudinal uniform distributed electron beam with current of $I$ and rms transverse beam size of $\sigma$, the output power of a CHG with radiator length of $l_{r}$ can be simplified as [40]

$$
P_{\mathrm{coh}}=\frac{Z_{0}\left(K[J J]_{1} l_{r} I b_{n}\right)^{2}}{32 \pi \sigma^{2} \gamma^{2}}
$$

where $Z_{0}=377 \Omega$ is the vacuum impedance, $K$ is the dimensionless undulator parameter, and $[J J]_{1}$ is the polarization modification factor for a linearly polarized planar undulator. Here we assume that the bunching factor in the two integral gain length is constant for simply estimating the peak power in the short radiator. One can find from Eq. (2) that the output power of CHG is strongly coupled with the beam current, bunching factor, and transverse beam size but nearly independent of the initial beam energy spread, which means that a large energy modulation amplitude is acceptable in CHG. Therefore, the CHG scheme can be used to generate coherent signal at ultrahigh harmonics of the seed. Comparing with the conventional HGHG, the only drawback of CHG is the relative low output power. However, in the proposed scheme, the coherent signal from $\mathrm{CHG}$ can be further amplified by the fresh parts of the electron beam in the following undulator sections. As shown in Fig. 1(c), the coherent signal is shifted forward by a small delay chicane inserted between the undulator sections. The microbunchings formed in the previous undulator section are smeared out by this chicane, preventing the growth of noisy spikes in the following undulators. The coherent signal reseeds the fresh bunch in the following undulator, leading to an amplification of the radiation pulse until saturation. This procedure is repeated in the following chicane-undulator (C-U) modules, as shown in Fig. 1(d). The final output power of the radiation pulse can be much higher than the saturation power of a conventional seeded FEL at the same wavelength.

It is anticipated that seed FELs can generate a nearly transform-limited radiation pulse, which usually requires a uniform electron beam with nearly constant energy and current distributions. However, the energy profile of the electron beam usually has an initial energy curvature due to the radio frequency curvature, wakefield effects, and microbunching instabilities in the linac. Generally, this energy curvature will impact the FEL gain process and result in a broader output spectral bandwidth $[41,42]$. When compared with the conventional cascaded HGHG scheme, the proposed HB-HGHG scheme only needs one DS with much smaller dispersion strength due to the relatively large energy modulation amplitude. This means that the effects of the imperfect energy profile on the output coherence can be significantly reduced. This is another advantage of the HB-HGHG for generating ultrahigh harmonic radiation pulses.
TABLE I. Main parameters of the SXFEL user facility.

\begin{tabular}{lc}
\hline \hline Electron beam & \\
Energy & $1.6 \mathrm{GeV}$ \\
Bunch charge & $500 \mathrm{pC}$ \\
Peak current & $900 \mathrm{~A}$ \\
Energy spread & $160 \mathrm{keV}$ \\
Normalized emittance & $0.6 \mathrm{~mm} \cdot \mathrm{mrad}$ \\
Full bunch length & $600 \mathrm{fs}$ \\
Seeding laser & \\
Wavelength & $265 \mathrm{~nm}$ \\
Peak power & $4 \mathrm{GW}$ \\
Pulse length (FWHM) & $30 \mathrm{fs}$ \\
Rayleigh length & $2.96 \mathrm{~m}$ \\
Modulator & \\
Period & $8 \mathrm{~cm}$ \\
Number of Periods & 20 \\
$K$ & 11.02 \\
Dispersion section & \\
$R_{56}$ & \\
$R 1$ & $12 \mu \mathrm{m}$ \\
Period & \\
Peak field & $2.35 \mathrm{~cm}$ \\
Radiation wavelength & 2.24 \\
Radiator length & $1.019 \mathrm{~T}$ \\
\hline \hline
\end{tabular}

To show the principle and limits of the HB-HGHG, here we adopt the nominal parameters of the SXFEL user facility, as shown in Table I, to carry out some theoretical calculations. The SXFEL user facility is initially designed for generating coherent soft x-ray pulses in the "water window" (the wavelength of electromagnetic spectrum range from $2.34 \mathrm{~nm}-4.4 \mathrm{~nm}$ ) with a $1.6 \mathrm{GeV}$ electron beam. The baseline of SXFEL is a two-stage HGHG with a UV seed laser at $265 \mathrm{~nm}$. The total harmonic up-conversion number for the two stages is $12 \times 5$. By using the proposed HB-HGHG scheme, this task could be performed with a single stage setup.

With the electron beam and radiator parameters listed in Table I, the Pierce parameter of the FEL at $4.4 \mathrm{~nm}$ is calculated to be about $\rho_{r}=1.3 \times 10^{-3}$. Since the initial relative energy spread of the electron beam is about $1 \times 10^{-4}$, the maximal laser induced energy spread can be only about 10 times larger than the initial energy spread, which means that the harmonic up-conversion number of a conventional HGHG is limited to about 10. By using the HB-HGHG scheme, the harmonic up-conversion number can be much higher. The maximal peak power of the $265 \mathrm{~nm}$ seed laser in the modulator of SXFEL is about $4 \mathrm{GW}$. By fully using this high peak power, the energy modulation amplitude can be increased to about 38 times of the initial beam energy spread. With this energy modulation amplitude, Fig. 2 shows the analytical calculation results of the bunching factors at the entrance of the radiator. One can 


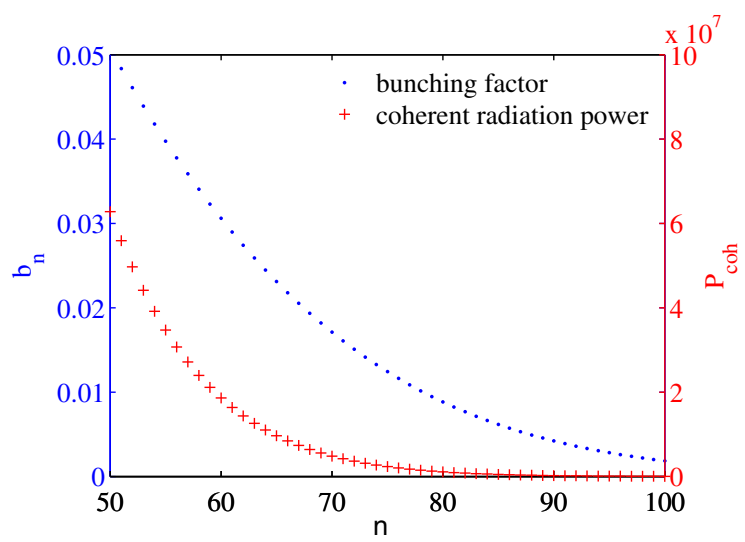

FIG. 2. The bunching factor (blue dot) and coherent radiation power (red cross) of the $\mathrm{CHG}$ as a function of the harmonic number.

find that the bunching factor at 60th harmonic of the seed is about 3\%, which is sufficient for initiating the coherent radiation in the following radiator. According to Eq. (2), the peak power of the coherent signal from $R 1$ is calculated to be about $20 \mathrm{MW}$, which will be amplified by the following $\mathrm{C}-\mathrm{U}$ modules.

\section{REQUIREMENTS FOR THE SEED LASER AND DELAY CHICANES}

The main purpose of the proposed technique is to produce fully coherent $\mathrm{x}$-ray pulses with very high peak power. However, the harmonic multiplication process also amplifies the small phase errors of the seed laser and the initial shot noise of the electron beam. These effects may overwhelm the external seeding source at ultrahigh harmonics and impede the generation of transform-limited pulses. The effect from imperfection of the seed laser can be mitigated by the phase smoothing effect due to the FEL slippage effect in the modulator [43]. For a $30 \mathrm{fs}$ seed laser pulse at $265 \mathrm{~nm}$, a modulator with period number of 20 allows one to preserve the good temporal coherence in the presence of phase errors in the seed laser. It has been theoretically demonstrated that the time-bandwidth product factor of the 30th harmonic radiation can be smaller than 1.5 times of that of a Fourier transform-limit pulse. By adopting a modulator that is resonant at an odd subharmonic of the seed laser, the time-bandwidth product factor of the high harmonic radiation can be close to that of a Fourier transform-limit pulse [43]. For the 60th harmonic radiation in the water window, the initial phase error in the seed laser would not be a serious problem for the HB-HGHG scheme.

To suppress the electron beam shot noise effect and get sufficient contrast in the HB-HGHG, the initial seed laser power $P_{\text {seed }}$ should be much higher than the equivalent shot noise power in the modulator, which can be estimated by $[44,45]$

$$
P_{\text {noise }}=\frac{3^{3 / 4} 4 \pi \rho_{m}^{2} P_{\text {beam }}}{N_{\lambda s} \sqrt{\pi l_{m} / L_{g m}}},
$$

where $P_{\text {beam }}$ is the electron beam power, $N_{\lambda s}$ is the number of electrons per seed laser wavelength, $l_{m}$ is the modulator length, $\rho_{m}$ and $L_{g m}$ are the Pierce parameter and FEL gain length in the modulator, respectively. To suppress the shot noise, the following requirement should be satisfied [46]:

$$
P_{\text {seed }} / P_{\text {noise }} \gg n^{2} \text {. }
$$

Using the parameters listed in Table I, the shot noise power is calculated to be about $110 \mathrm{~W}$. The maximal seed laser power is about $3.6 \times 10^{7}$ times larger than the shot noise power. The requirement of Eq. (4) can be easily satisfied for a soft x-ray FEL in the water window since the required harmonic number is lower than 130 .

The degradation of the output coherence of the HB-HGHG may also arise from the signal-to-noise ratio of the radiation pulse from the $\mathrm{CHG}$, which acts as a startup seed for the following chicane-undulator modules. The power of the radiation that starts from electron beam shot noise can be estimated by [47]

$$
P_{\text {out }} \approx \frac{1}{9} P_{s t} \exp \left(l_{r} / L_{g r}\right),
$$

where $P_{s t}$ is the start-up shot noise power and $L_{g r}$ is the gain length of the SASE-FEL in $R 1$. The output power of CHG can be estimated by Eq. (2). The constraint on the signal-to-noise ratio of the radiation pulse from $R 1$ is

$$
P_{\text {coh }} / P_{\text {out }} \gg 1
$$

With the parameters listed in Table I, Fig. 3 shows the calculation results of coherent radiation power and noise power for various high harmonic numbers $(>60)$ using

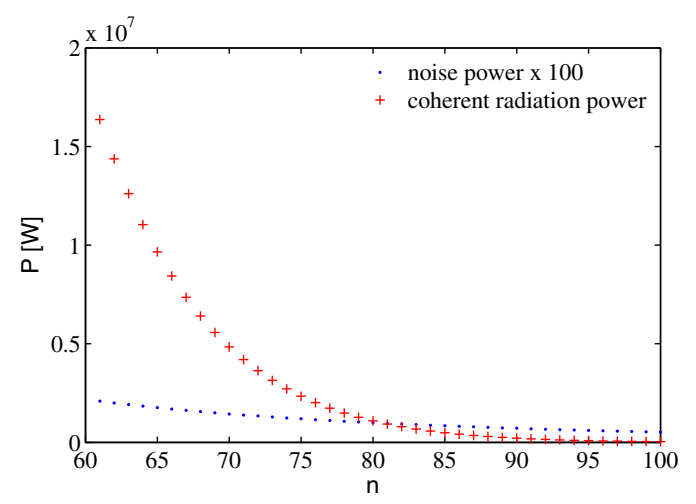

FIG. 3. Comparison of the radiation power of $\mathrm{CHG}$ (red cross) and the noise power (blue dot) from $R 1$ for different harmonic numbers. 


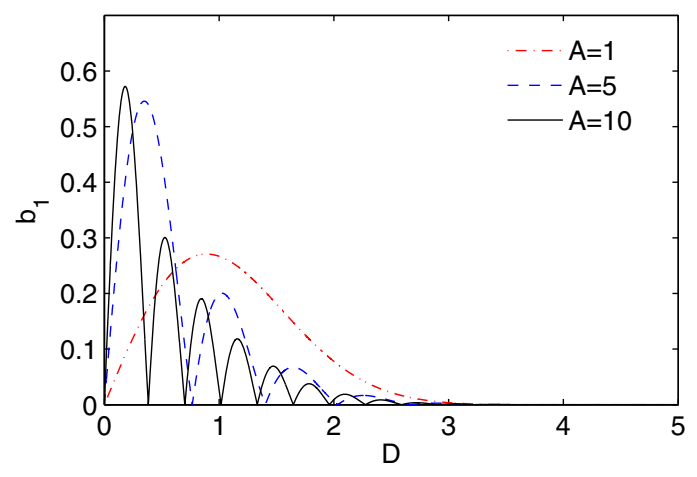

FIG. 4. The fundamental bunching factors as a function of the delay chicane strengths for different energy modulation amplitudes.

Eq. (2) and Eq. (5). One can find that the coherent radiation power at 60th harmonic of the seed is about 3 orders of magnitude higher than the noise power. However, the signal to noise ratio drops quickly at higher harmonics due to the relatively small bunching factor. The power of the coherent signal may be not sufficient to dominate the FEL gain process in $R 1$ for the harmonic number larger than 80 . However, it is worth pointing out here that the output power of CHG is proportional to the square of $b_{n}$. A larger $\Delta \gamma$ will help one to further enhance the output intensity of CHG. It is still possible to extend the short wavelength cover range of a single stage HB-HGHG with higher seed laser power.

Besides the shot noise, the spectral noise may also come from the disturbed parts of the electron beam that have already lased in the previous undulator sections. These parts of electron beam contain considerable coherent microbunchings and may generate powerful noisy spikes in the following undulators. One of the important functions of the delay chicane is to smear out these microbunchings. The bunching factor at the radiation wavelength as a function of the delay chicane strength can also be calculated by Eq. (1) with $k_{s}$ replaced by $k_{r}$, which is the wave number of the radiation. The calculation results are illustrated in Fig. 4. One can find that the bunching factors oscillate as the dispersion strengths are increased. For a relative large $D$, the bunching factors will drop quickly, e.g. for $D=4$, all the bunching factors drop below $5 \times 10^{-5}$, which is at the shot noise level. To suppress the noise from the disturbed part of the electron beam at SXFEL, the required $R_{56}$ of the delay chicanes should be larger than $28 \mu \mathrm{m}$ (equivalent to $D=4$ ).

\section{START-TO-END SIMULATIONS FOR HB-HGHG}

To illustrate the possible performance with realistic parameters and show the optimization method of the HB-HGHG, we carried out start-to-end simulations with the nominal parameters of the SXFEL user facility, as shown in Table I. The schematic layout of the SXFEL is shown in Fig. 5. The linac of SXFEL consists of a photoinjector, a laser heater system (LH), an X-band linearizer, S-band and C-band accelerating structures, and two magnetic bunch compressors (BCs). The undulator system is designed for HB-HGHG, which consists of a modulator, a dispersion chicane, a short radiator $(R 1)$ and 5 C-U modules.

The simulation of the electron beam dynamics in the photoinjector was performed by ASTRA [48], which takes into account space-charge effects. The electron beam with peak current of about $30 \mathrm{~A}$ is accelerated to about $130 \mathrm{MeV}$ at the end of the injector. elegant [49] was then used for the simulation in the remainder of the linac. The uncorrelated energy spread of the electron beam was increased from about $1 \mathrm{keV}$ to about $5 \mathrm{keV}$ by the $\mathrm{LH}$ to suppress the microbunching instability in the linac. An X-band linearizer is employed before the first bunch compressor to compensate the second order nonlinear components in the longitudinal phase space to avoid the undesired growth of the transverse emittance and to produce a more flat current profile. Simulation results for the longitudinal phase space and the current profile of the electron beam at the exit of the linac are shown in Fig. 6, where one can find a peak current of about $900 \mathrm{~A}$ is achieved and a constant profile is maintained in the approximately $t_{\text {con }}=300 \mathrm{fs}$ wide region. This part of electron beam is used for the coherent FEL pulse generation.
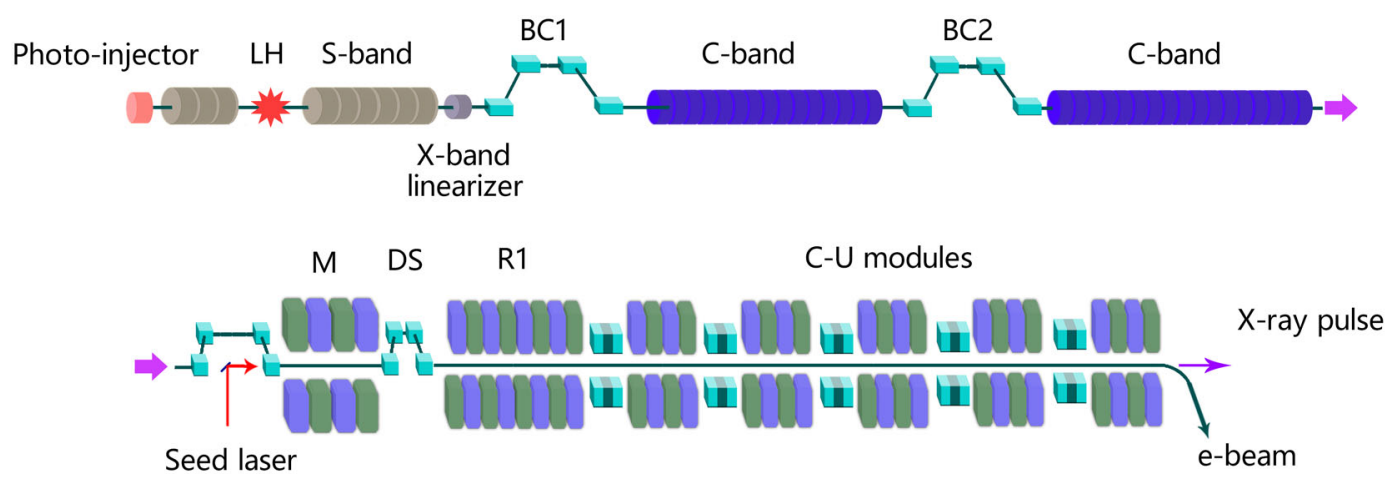

FIG. 5. Layout of the SXFEL. 

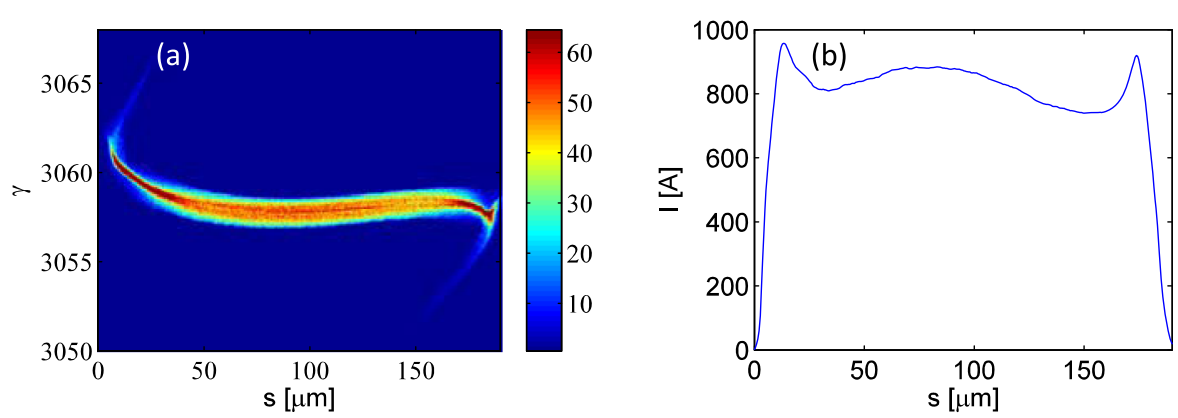

FIG. 6. Simulation results for (a) the longitudinal phase and (b) current profile of the electron beam at the entrance of the HB-HGHG scheme.

The modulation and FEL gain processes were simulated by genesis [50] based on the output of elegant. A UV seed laser at $265 \mathrm{~nm}$ with pulse duration of about $30 \mathrm{fs}$ (FWHM) is adopted to interact with the tail part of the electron beam in a modulator with 20 periods with a length of $8 \mathrm{~cm}$ each. The seed laser peak power is about $4 \mathrm{GW}$ with an rms stability of about $1.5 \%$, which is smaller than that of FERMI FEL [37] and thus can guarantee a relative stable CHG output. The longitudinal phase of the seed laser is nearly invariable from shot to shot. The laser induced energy modulation amplitude is about $6.1 \mathrm{MeV}$, which is about 38 times of the uncorrelated beam energy spread. A dispersion section with $R_{56}$ of about $12 \mu \mathrm{m}$ is employed to convert energy modulation into density modulation. The simulation results for the longitudinal phase space and corresponding bunching factor distribution along the electron bunch at the entrance of $R 1$ are shown in Figs. 7(a) and 7(b). The bunching factor at 60th harmonic of the seed is over $2.7 \%$, which is responsible for the initial steep quadratic growth of the radiation power in $R 1$, as shown in Fig. 7(c). The output peak power of CHG at $4.4 \mathrm{~nm}$ is about $18 \mathrm{MW}$, which fits quite well with the analytical calculation result of Eq. (2). The output pulse duration is about $20 \mathrm{fs}$ (FWHM), which is a little shorter than the seed laser pulse length due to the pulse shorten effect in the seeded FEL [50].
The CHG signal is then shifted forward to the fresh part of the electron beam and sent into the downstream $\mathrm{C}-\mathrm{U}$ modules to get further amplification. The dispersion strengths of the delay chicanes should be large enough to smear out the microbunchings formed in the previous undulator sections and the time delays induced by the chicanes should be larger than the seed laser pulse length. The $R_{56} \mathrm{~S}$ of the delay chicanes are all set to be $36 \mu \mathrm{m}$, which can introduce a dispersion strength of $D \approx 5.14$ and a relative time delay of about $t_{\text {delay }}=60 \mathrm{fs}$. Considering that the radiation from $\mathrm{CHG}$ is quite weak, a relative long undulator section with length of about $8 \mathrm{~m}$ is adopted in the first C-U module to fully amplify the CHG signal. The lengths of the rest C-U modules are all set to be about $4.8 \mathrm{~m}$, which is long enough to place a $4.2 \mathrm{~m}$ long undulator and a $0.48 \mathrm{~m}$ long delay chicane. The delay chicane consists of three dipole magnets. The lengths of the first and third dipoles are $7 \mathrm{~cm}$. The length of the second dipole is $14 \mathrm{~cm}$. The distance between the first two dipoles is $10 \mathrm{~cm}$. The required bending angles of the first and third dipoles are about $11.1 \mathrm{mrad}$. The peak field of the undulators in the $\mathrm{C}-\mathrm{U}$ modules are all chosen to be $1.016 \mathrm{~T}$ in the simulation to optimize the FEL output power. The simulation results for the FEL gain curve and the radiation pulse evolutions in the $\mathrm{C}-\mathrm{U}$ modules are summarized in Fig. 8. The peak power from the first
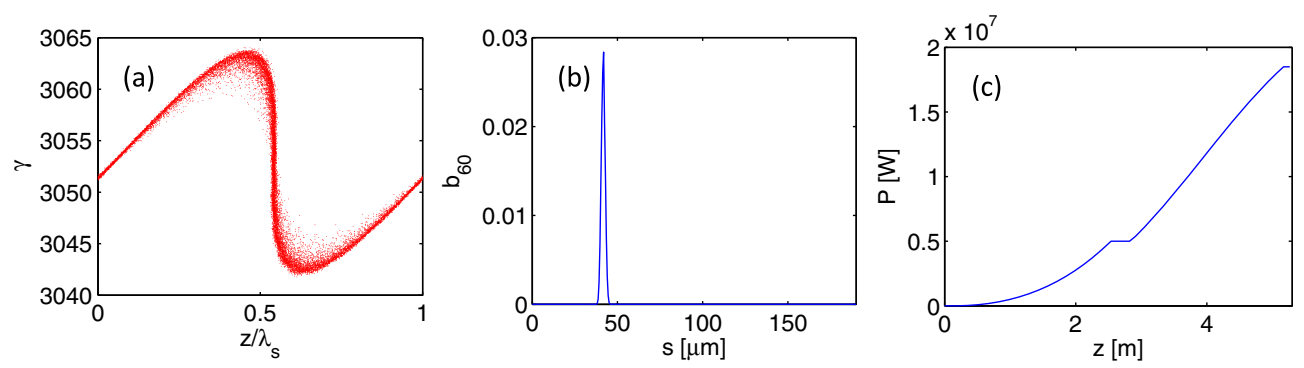

FIG. 7. Simulation results for the CHG: (a) Longitudinal phase space in one seed laser wavelength region at the entrance of $R 1$; (b) the 60th harmonic bunching factor distribution along the electron bunch at the entrance of $R 1$; (c) the gain curve of the FEL peak power in the $R 1$. 

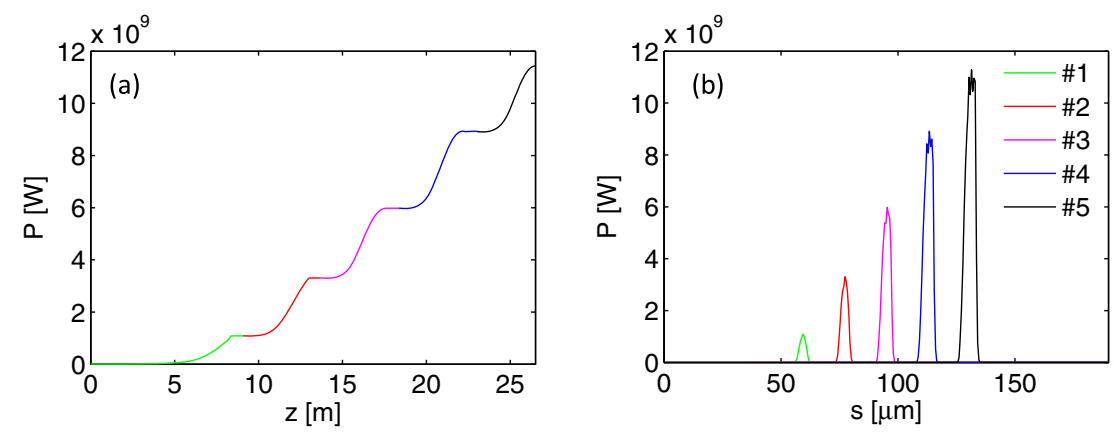

FIG. 8. The FEL gain curve (a) and structure evolutions of the radiation pulse (b) in the C-U modules.

undulator section is about $1.6 \mathrm{GW}$, which is already comparable to the saturation power of the single-stage EEHG FEL or a two-stage cascaded HGHG FEL at the same wavelength [51,52]. This intense radiation pulse can be further amplified by the following $\mathrm{C}-\mathrm{U}$ modules via the superradiant process. As shown in Fig. 8, the peak power grows quadratically in each undulator section and gets a nearly same power gain of about $2.6 \mathrm{GW}$ at the end of each $\mathrm{C}-\mathrm{U}$ module. As a result, the radiation peak power increases almost linearly with the number of $\mathrm{C}-\mathrm{U}$ modules. The pulse length of about $20 \mathrm{fs}$ (FWHM) is maintained through the amplification processes. After $5 \mathrm{C}-\mathrm{U}$ modules, the peak power is increased to about $11.5 \mathrm{GW}$. The maximal output peak power of HB-HGHG is mainly limited by $t_{\text {con }} / t_{\text {delay }}$, which determines the available shift times in the C-U modules. For the SXFEL, the maximal number of shift times is about 5. By using a longer electron beam or a shorter seed laser pulse, the output peak power can be further enhanced.

The simulation result for the final output spectrum is shown in Fig. 9. The FWHM bandwidth of the spectrum is about $0.09 \%$, which is about 1.4 times of the Fourier transform limit. The small broadening of the bandwidth is mainly caused by the nonlinear energy chirp in the electron beam.

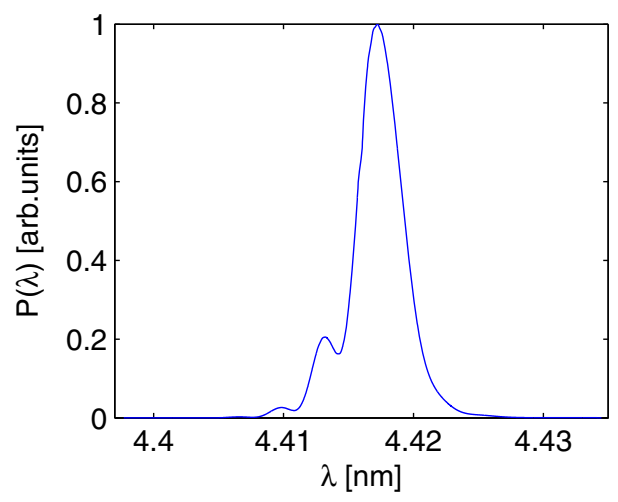

FIG. 9. The output spectrum of the HB-HGHG.

\section{CONCLUSIONS}

In conclusion, the HB-HGHG scheme has been proposed in this paper for enhancing the harmonic up-conversion efficiency and the output intensity of a seeded FEL. The proposed scheme is simple and easy to be implemented at seeded FEL facilities. In addition to a standard HGHG scheme, it only needs small delay chicanes between undulator sections. Some practical limiting factors that might affect the performance of the proposed scheme are also taken into account, and it is shown that with the present technologies, these effects can be controlled within an acceptable level. The feasibility of operating HB-HGHG for the generation of high brightness and coherent radiation pulses in the water window has been demonstrated by numerical simulations. The simulation results show that the proposed scheme can enhance the peak power by about one order of magnitude with respect to a conventional HGHG. The output peak power can be further increased by using more C-U modules and a longer electron beam. The short-wavelength cover range can also be extended by using a seed laser pulse with higher peak power. This kind of light source would allow one to perform many challenging experiments which require narrow bandwidth soft $\mathrm{X}$-ray radiation pulses.

\section{ACKNOWLEDGMENTS}

The authors would like to thank Dazhang Huang, Zhen Wang, Zheng Qi, and Kaiqing Zhang for helpful discussions and useful comments. This work is supported by the National Natural Science Foundation of China (11475250) and Youth Innovation Promotion Association CAS.

[1] W. A. Ackermann et al., Operation of a free-electron laser from the extreme ultraviolet to the water window, Nat. Photonics 1, 336 (2007).

[2] P. Emma et al., First lasing and operation of an ångstromwavelength free-electron laser, Nat. Photonics 4, 641 (2010). 
[3] T. Ishikawa et al., A compact X-ray free-electron laser emitting in the sub-ångstrom region, Nat. Photonics 6, 540 (2012).

[4] M. Altarelli et al., DESY, Technical Design Report DESY 2006-097, 2007.

[5] R. Ganter et al., SwissFEL conceptual design report, PSI, 2010.

[6] J. H. Han, H. S. Kang, and I. S. Ko, in Proceedings of the 3rd International Particle Accelerator Conference, New Orleans, LA, 2012 (IEEE, Piscataway, NJ, 2012), p. 1735.

[7] A. Kondratenko and E. Saldin, Generation of coherent radiation by a relativistic-electron beam in an undulator, Part. Accel. 10, 207 (1980).

[8] R. Bonifacio, C. Pellegrini, and L. M. Narducci, Collective instabilities and high-gain regime in a free electron laser, Opt. Commun. 50, 373 (1984).

[9] T. Winzer, A. Knorr, M. Mittendorff, S. Winnerl, M. Lien, D. Sun, T. B. Norris, M. Helm, and E. Malic, Absorption saturation in optically excited graphene, Appl. Phys. Lett. 101, 221115 (2012).

[10] J. D. Biggs, Y. Zhang, D. Healion, and S. Mukamela, Twodimensional stimulated resonance Raman spectroscopy of molecules with broadband x-ray pulses, J. Chem. Phys. 136, 174117 (2012).

[11] K. C. Prince et al., Coherent control with a shortwavelength free-electron laser, Nat. Photonics 10, 176 (2016).

[12] F. Bencivenga et al., Four-wave mixing experiments with extreme ultraviolet transient gratings, Nature (London) 520, 205 (2015).

[13] J. Feldhaus and E. L. Saldin, Possible application of X-ray optical elements for reducing the spectral bandwidth of an X-ray SASE FEL, Opt. Commun. 140, 341 (1997).

[14] G. Geloni, V. Kocharyan, and E. L. Saldin, A novel selfseeding scheme for hard X-ray FELs, J. Mod. Opt. 58, 1391 (2011).

[15] J. Amann et al., Demonstration of self-seeding in a hardX-ray free-electron laser, Nat. Photonics 6, 693 (2012).

[16] D. Ratner et al., Experimental Demonstration of a Soft X-Ray Self-Seeded Free-Electron Laser, Phys. Rev. Lett. 114, 054801 (2015).

[17] J. Wu, A. Marinelli, and C. Pellegrini, in Proceedings of the 34th International Free Electron Laser Conference (FEL12), Nara, Japan, 2012 (JACoW, Nara, Japan, 2012), p. 237.

[18] D. Xiang, Y. Ding, Z. Huang, and H. Deng, Purified Selfamplified Spontaneous Emission (pSASE) Free-electron Lasers with Slippage-boosted Filtering, Phys. Rev. ST Accel. Beams 16, 010703 (2013).

[19] J. Wu et al., in Proceedings of the 4th International Particle Accelerator Conference, IPAC-2013, Shanghai, China (JACoW, Shanghai, China, 2013), p. 2068.

[20] B. W. J. McNeil, N. R. Thompson, and D. J. Dunning, Transform-Limited X-Ray Pulse Generation From a High-Brightness Self-Amplified Spontaneous-Emission Free-Electron Laser, Phys. Rev. Lett. 110, 134802 (2013).

[21] L. H. Yu, Generation of intense uv radiation by subharmonically seeded single-pass free-electron lasers, Phys. Rev. A 44, 5178 (1991).
[22] G. Stupakov, Using the Beam-Echo Effect for Generation of Short-Wavelength Radiation, Phys. Rev. Lett. 102, 074801 (2009).

[23] D. Xiang and G. Stupakov, Echo-enabled harmonic generation free electron laser, Phys. Rev. ST Accel. Beams 12, 030702 (2009).

[24] H. Deng and C. Feng, Using Off-Resonance Laser Modulation for Beam-Energy-Spread Cooling in Generation of Short-Wavelength Radiation, Phys. Rev. Lett. 111, 084801 (2013).

[25] C. Feng, H. Deng, D. Wang, and Z. Zhao, Phase-merging enhanced harmonic generation free-electron laser, New J. Phys. 16, 043021 (2014).

[26] E. Allaria et al., Highly coherent and stable pulses from the FERMI seeded free-electron laser in the extreme ultraviolet, Nat. Photonics 6, 699 (2012).

[27] B. Liu et al., Demonstration of a widely-tunable and fully-coherent high-gain harmonic-generation free-electron laser, Phys. Rev. ST Accel. Beams 16, 020704 (2013).

[28] E. Allaria et al., Two-stage seeded soft-X-ray free-electron laser, Nat. Photonics 7, 913 (2013).

[29] D. Xiang et al., Demonstration of the Echo-Enabled Harmonic Generation Technique for Short-Wavelength Seeded Free Electron Lasers, Phys. Rev. Lett. 105, 114801 (2010).

[30] Z. T. Zhao et al., First lasing of an echo-enabled harmonic generation free-electron laser, Nat. Photonics 6, 360 (2012).

[31] E. Hemsing, M. Dunning, B. Garcia, C. Hast, T. Raubenheimer, G. Stupakov, and D. Xiang, Echo-enabled harmonics up to the 75th order from precisely tailored electron beams, Nat. Photonics 10, 512 (2016).

[32] E. Prat, F. Löhl, and S. Reiche, Efficient generation of short and high-power x-ray free-electron-laser pulses based on superradiance with a transversely tilted beam, Phys. Rev. ST Accel. Beams 18, 100701 (2015).

[33] R. Bonifacio, N. Piovella, and B. W. J. McNeil, McNeil, Superradiant evolution of radiation pulses in a free-electron laser, Phys. Rev. A 44, R3441 (1991).

[34] T. Tanaka, Proposal for a Pulse-Compression Scheme in X-Ray Free-Electron Lasers to Generate a Multiterawatt, Attosecond X-Ray Pulse, Phys. Rev. Lett. 110, 084801 (2013).

[35] E. Prat and S. Reiche, Simple Method to Generate TerawattAttosecond X-Ray Free-Electron-Laser Pulses, Phys. Rev. Lett. 114, 244801 (2015).

[36] S. Huang, Y. Ding, Z. Huang, and G. Marcus, Generation of subterawatt-attosecond pulses in a soft x-ray free-electron laser, Phys. Rev. Accel. Beams 19, 080702 (2016).

[37] C. Bocchetta et al. (Fermi Collaboration), FERMI@Elettra Conceptual Design Report (Sincrotrone, Trieste, 2007).

[38] Z. T. Zhao, S. Y. Chen, L. H. Yu, C. X. Tang, L. X. Yin, D. Wang, and Q. Gu, in Proceedings of the 2nd International Particle Accelerator Conference, IPAC-2011, San Sebastián, Spain (EPS-AG, Spain, 2011), p. 3011.

[39] SXFEL user facility feasibility study report, 2016.

[40] Li. H. Yu and J. Wu, Theory of high gain harmonic generation: an analytical estimate, Nucl. Instrum. Methods Phys. Res., Sect. A 483, 493 (2002). 
[41] Z. Huang, D. Ratner, G. Stupakov, and D. Xiang, in Proceedings of the 31st International Free Electron Laser Conference (FEL 09), Liverpool, UK (STFC Daresbury Laboratory, Warrington, 2009), p. 127.

[42] C. Feng, D. Wang, and Z. Zhao, in Proceedings of the 3rd International Particle Accelerator Conference, New Orleans, LA, 2012 (IEEE, Piscataway, NJ, 2012), p. 1724.

[43] C. Feng, H. Deng, G. Wang, D. Wang, Z. Zhao, and D. Xiang, Slippage effect on energy modulation in seeded free-electron lasers with frequency chirped seed laser pulses, Phys. Rev. ST Accel. Beams 16, 060705 (2013).

[44] E. L. Saldin, E. A. Schneidmiller, and M. V. Yurkov, The Physics of Free Electron Lasers(Springer, Berlin, 2000), p. 364.

[45] A. Marinelli, R. Bonifacio, C. Vaccarezza, and G. R. M. Robb, HGHG schemes for short wavelengths, Nucl. Instrum. Methods Phys. Res., Sect. A 593, 35 (2008).

[46] E. L. Saldin, E. A. Schneidmiller, and M. V. Yurkov, Study of a noise degradation of amplification process in a multistage HGHG FEL, Opt. Commun. 202, 169 (2002).

[47] Z. Huang and K. J. Kim, Review of x-ray free-electron laser theory, Phys. Rev. ST Accel. Beams 10, 034801 (2007).

[48] K. Floettmann, ASTRA User's Manual, available at http:// www.desy.de/mpyflo/Astra_dokumentationS (1999).

[49] M. Borland, Simple method for particle tracking with coherent synchrotron radiation, Phys. Rev. ST Accel. Beams 4, 070701 (2001).

[50] S. Reiche, GENESIS 1.3: a fully 3D time-dependent FEL simulation code, Nucl. Instrum. Methods Phys. Res., Sect. A 429, 243 (1999).

[51] D. Ratner, A. Fry, G. Stupakov, and W. White, Laser phase errors in seeded free electron lasers, Phys. Rev. ST Accel. Beams 15, 030702 (2012).

[52] Z. T. Zhao, C. Feng, J. H. Chen, and Z. Wang, Two-beam based two-stage EEHG-FEL for coherent hard X-ray generation, Science bulletin 61, 720 (2016). 